\title{
Weak Unit Disk and Interval Representation of Graphs
}

\author{
M.J. Alam ${ }^{1}$, S.G. Kobourov ${ }^{1}$, S. Pupyrev ${ }^{1,2(\bowtie)}{ }^{\text {, and J. Toeniskoetter }}{ }^{1}$ \\ 1 Department of Computer Science, University of Arizona, Tucson, AZ, USA \\ spupyrev@gmail.com \\ 2 Institute of Mathematics and Computer Science, Ural Federal University, \\ Yekaterinburg, Russia
}

\begin{abstract}
We study a variant of intersection representations with unit balls: unit disks in the plane and unit intervals on the line. Given a planar graph and a bipartition of the edges of the graph into near and far edges, the goal is to represent the vertices of the graph by unit-size balls so that the balls for two adjacent vertices intersect if and only if the corresponding edge is near. We consider the problem in the plane and prove that it is NP-hard to decide whether such a representation exists for a given edgepartition. On the other hand, we show that series-parallel graphs (which include outerplanar graphs) admit such a representation with unit disks for any near/far bipartition of the edges. The unit-interval on the line variant is equivalent to threshold graph coloring, in which context it is known that there exist girth-3 planar graphs (even outerplanar graphs) that do not admit such coloring. We extend this result to girth-4 planar graphs. On the other hand, we show that all triangle-free outerplanar graphs and all planar graphs with maximum average degree less than $26 / 11$ have such a coloring, via unit-interval intersection representation on the line. This gives a simple proof that all planar graphs with girth at least 13 have a unit-interval intersection representation on the line.
\end{abstract}

\section{Introduction}

Intersection graphs of various geometric objects have been extensively studied for their many applications [17]. A graph is a $d$-dimensional unit ball graph if its vertices are represented by unit-size balls in $\mathbb{R}^{d}$, and an edge exists between two vertices if and only if the corresponding balls intersect. Unit ball graphs are called unit disk graphs when $d=2$ and unit interval graphs when $d=1$. In this paper we study weak unit ball graphs: given a graph $G$ whose edges have been partitioned into "near" and "far" sets, we wish to assign unit balls to the vertices of $G$ so that, for an edge $(u, v)$ of $G$, the balls representing $u$ and $v$ intersect if the edge $(u, v)$ is near and do not intersect if the edge $(u, v)$ is far. Note that if $(u, v)$ is not an edge of $G$, then the balls of $u$ and $v$ may or may not intersect. We refer to such graphs as weak unit disk $(d=2)$ and weak unit interval graphs $(d=1)$. A geometric representation of such graphs (particularly, a mapping of the vertices to unit balls in $\mathbb{R}^{2}$ or $\mathbb{R}$ ), is called a weak unit disk representation 
or a weak unit interval representation; see Fig. 1. Near edges are shown as thick line segments and far edges are dashed line segments and we use this convention to distinguish near/far edges in the rest of the paper. Unit disk representations allow us to represent the edges of a graph by spatial proximity, which is intuitive from the point of view of human perception. Weak unit disk graphs also allow to arbitrarily forbid edges between certain pairs of vertices, which is useful in representation of "almost" unit disk graphs. It has been shown that weak unit interval graphs can be used to compute unit-cube contact representations of planar graphs $[5,18]$.

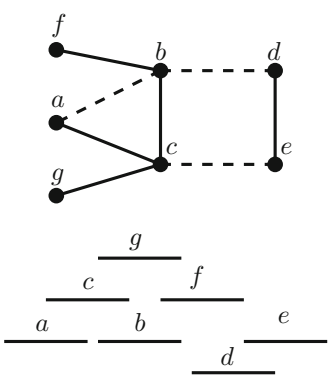

(a)

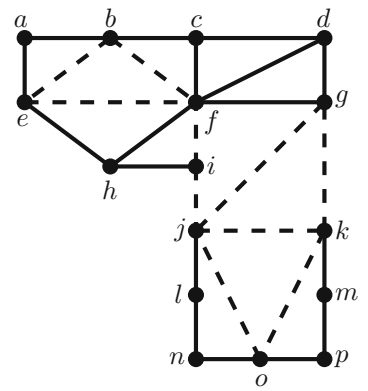

(b)

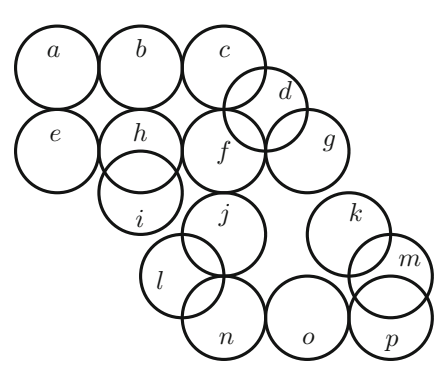

(c)

Fig. 1. (a) A graph with an edge-labeling and its weak unit interval representation. (b, c) A graph with an edge-labeling and its weak unit disk representation. In the figures we indicate near edges with solid lines and far edges with dashed lines.

Unit disk graphs have been extensively studied for their application to wireless sensor and radio networks. In such a network each sensor or radio can be modeled as a device with a unit size broadcast range, which naturally induces a unit disk graph by adding an edge whenever two ranges intersect. This setting makes it easy to study various practical problems. For example, in the frequency assignment problem the goal is to assign frequencies to radio towers so that nearby towers do not interfere with each other [15]. A weakness of the unit disk model is that it does not allow for interference between nodes (e.g., due to geography) and it does not account for the possibility that a pair of nodes may not be able to communicate (e.g., due to technological barriers). One attempt to address this issue are quasi unit disk graphs [19], where each vertex is represented by a pair of concentric disks, one of radius $r, 0<r<1$, and the other of radius 1 . In this model, two vertices are connected by an edge if their radius- $r$ disks overlap, and do not have an edge if their radius-1 disks do not overlap. The remaining edges are in or out of the graph on a case by case basis. In the weak unit disk model such problems can be dealt with by simply deleting edges between nodes which are nearby but whose ranges do not overlap (e.g., because they are separated by a mountain range). This gives us more flexibility than quasi unit disk graphs. 
Formally, an edge-labeling of a graph $G=(V, E)$ is a map $\ell: E \rightarrow\{N, F\}$. If $(u, v) \in E$, then $(u, v)$ is called near if $\ell(u, v)=N$, and otherwise $(u, v)$ is called far. In a unit disk (interval) representation $I$, each vertex $v \in V$ is represented as a disk (interval) centered at the point $I(v) \in \mathbb{R}^{2}(\mathbb{R})$. We denote by $\|I(u)-I(v)\|$ the distance between the points $I(u)$ and $I(v)$, and by a slight abuse of notation, we also refer to $I(v)$ as the disk (interval) representing $v \in V$. A weak unit disk (interval) representation of $G$ with respect to $\ell$ is a representation $I$ such that for each edge $(u, v) \in E,\|I(u)-I(v)\| \leq t$ if and only if $\ell(u, v)=N$, for some fixed unit $t>0$ (in other words, the disks and intervals have diameter $t$ ). Unless otherwise stated, we assume $t=1$. We say that a graph is a total weak unit disk (interval) graphs if it has an appropriate representation for all possible edge-labelings.

Related Work: Weak unit ball graphs can be seen as a form of graph drawing/labeling where a notion of "closeness" between vertices is used to define edges, from a given set of permissible edges. There are many classes of graphs defined on some notion of vertex closeness. For example, proximity graphs are those that can be drawn in the plane such that every pair of adjacent vertices satisfies some fixed notion of closeness, whereas every pair of non-adjacent vertices satisfy some notion of farness [20]. Examples of proximity graphs are Gabriel graphs, Delaunay triangulations, and relative neighborhood graphs. Gabriel graphs, defined in the context of categorizing biological populations [13], can be embedded in the plane so that for every pair of vertices $(u, v)$, the disk with $u$ and $v$ as antipodal points contains no other vertex if and only if $(u, v)$ is an edge. Recently, Evans et al. [10] studied region of influence graphs, where each pair of vertices $u, v$ in the plane is assigned a region $R(u, v)$, and there is an edge if and only if $R(u, v)$ contains no vertices, except possibly $u$ and $v$. They generalize this class of graphs to approximate proximity graphs, where there are parameters $\epsilon_{1}>0$ and $\epsilon_{2}>0$, such that a vertex other than $u$ or $v$ is contained in $R(u, v)$, scaled by $1 /\left(1+\epsilon_{1}\right)$, if and only if $(u, v)$ is an edge; the region $R(u, v)$, scaled by $1+\epsilon_{2}$, is empty if and only if $(u, v)$ is not an edge. However there is a significant difference between the notion of proximity graphs and the notion of weak unit ball graphs. In proximity graphs the notion of closeness is defined by two groups, namely adjacent and non-adjacent pairs of vertices, whereas for weak unit ball graphs, there are three groups. Specifically, the near and far edges in the input graph $G$ represent vertex pairs with closeness and farness requirements, while all nonadjacent vertex pairs in $G$ have no requirement on proximity. Thus proximity graphs is more restricted than the weak unit ball graphs, in that they can be modeled by weak unit ball graphs where the input graph is the complete graph $K_{n}$.

Weak unit ball representability in $1 \mathrm{D}$ is related to the recently introduced threshold-coloring problem [1] and we show that these two problems are in fact equivalent. In this variant of graph coloring, integer colors are assigned to the vertices so that endpoints of near edges differ by less than a given threshold, while endpoints of far edges differ by more than the threshold. Deciding whether a graph is threshold-colorable with respect to a given partition of edges into 
near and far is equivalent to the graph sandwich problem for unit-intervalrepresentability, which is known to be NP-hard [14]. Hence, deciding whether a graph admits a weak unit interval representation with respect to a given edgelabeling is also NP-hard. In fact, along the lines of argument used in [1], one can prove that recognizing weak unit ball graphs with a given edge-labeling in any dimension $d=1,2, \ldots$ is equivalent to the graph sandwich problem for unit-ballrepresentability in dimension $d$. Note that the problem of recognizing weak unit interval graphs is different than recognizing unit interval graphs, which can be done in linear time [11]. It is known that planar graphs with girth (the length of a shortest cycle in the graph) at least 10 are always threshold-colorable. Several Archimedean lattices (which correspond to tilings of the plane by regular polygons), and some of their duals, the Laves lattices, are also threshold-colorable [2] for any edge-labeling. Hence, these graph classes are weak unit interval graphs.

Unit interval graphs are also related to threshold and difference graphs. In threshold graphs there exists a real number $S$ and for every vertex $v$ there is a real weight $a_{v}$ so that $(v, w)$ is an edge if and only if $a_{v}+a_{w} \geq S$ [21]. A graph is a difference graph if there is a real number $S$ and for every vertex $v$ there is a real weight $a_{v}$ so that $\left|a_{v}\right|<S$ and $(v, w)$ is an edge if and only if $\left|a_{v}-a_{w}\right| \geq S$ [16]. Note that for both these classes the existence of an edge is completely determined by the threshold $S$, while in our setting the edges defined by the threshold (size of the ball) must also belong to the original (not necessarily complete) graph. Threshold-colorability is also related to the integer distance graph representation [9,12]. An integer distance graph is a graph with the set of integers as vertex set and with an edge joining two vertices $u$ and $v$ if and only if $|u-v| \in D$, where $D$ is a subset of the positive integers.

Our Results: We introduce the notion of weak unit disk and interval representations. While finding representations with unit intervals is equivalent to threshold-coloring where some results are already known, the problem of weak unit disk representability is new. We first show that recognizing weak unit disk graphs is NP-hard. Note that the NP-hardness of the unit interval variant follows from the results in [1].

We then consider subclasses of planar graphs that admit weak unit disk (interval) representation. We show that every 2-reducible graph (as defined later) has a weak unit disk representation for any edge-labeling. In particular, any series-parallel graph (which includes all outerplanar graphs) has a weak unit disk representation for any edge-labeling. For representation with unit intervals, it follows from [1] that all planar graphs with girth at least 10 are total weak unit interval graphs. We generalize the result by proving that graphs of bounded maximum average degree have weak unit interval representations for any given edge-labeling. In the other direction, we construct an example of a planar girth4 graph which is not a total weak unit interval graph, improving on the earlier girth-3 example. Further, we show that dense planar graphs do not always admit weak unit interval graph representation.

Finally we study outerplanar graphs. It is known that some outerplanar graphs with girth 3 are not total weak unit interval graphs, and our example 
of girth-4 graph is not outerplanar. Thus, a natural question in this context is whether every girth-4 outerplanar graph admits weak unit interval representation for any edge-labeling. We show that this is indeed the case.

\section{Weak Unit Disk Graph Representations}

First we consider the complexity of recognizing weak unit disk graphs.

Lemma 1. It is NP-hard to decide if a graph $G$ with an edge-labeling $\ell$ admits a weak unit disk representation, even if the edges labeled $N$ induce a planar subgraph.

Proof. It is known that deciding whether a planar graph is a unit disk graph is NP-hard [6]. Let $n$ be the number of vertices of $G$, and define an edge-labeling $\ell$ of $K_{n}$ by setting $\ell(e)=N$ if and only if $e$ is an edge of $G$. Clearly, a unit disk representation of $G$ is also a weak unit disk representation of $K_{n}$ with respect to $\ell$ and vice versa.

Note that Lemma 1 only proves NP-hardness, and the problem of deciding whether a graph with an edge-labeling has a weak unit disk representation is not known to be in NP. The obvious approach is to use a weak unit disk representation as a polynomial size certificate. Unfortunately, it has recently been showed that unit disks graphs on $n$ vertices may require $2^{2^{\Theta(n)}}$ bits for a unit disk representation with integer coordinates [22].

\section{Unit Disk Representation of Outerplanar and Related Graphs}

Note that the class of weak unit disk graphs strictly contains the class of weak unit interval graphs. For example, in Fig. 2, we provide a weak unit disk representation of the sungraph for a particular edge-labeling, which does not admit a weak unit interval representation. Our main goal here is to prove that every series-parallel graph is a total weak unit disk graph. To this end, we study a larger class of graphs, called 2-reducible graphs [25]. A simple graph $G$ is a 2-reducible graph if one of the following holds:

1. $G$ is an independent set;

2. $G$ has an edge $(u, v)$ such that $v$ has degree at most 2 , and the graph obtained by contracting $(u, v)$ and removing parallel edges is a 2-reducible graph.

Note that 2-reducible graphs are a subclass of 2-degenerate graphs, which are graphs where every subgraph has a vertex with degree at most 2 [23]. For example, the graph obtained by subdividing one edge of $K_{4}$ is a 2-degenerate graph, but not a 2-reducible graph.

Theorem 1. Every 2-reducible graph is a total weak unit disk graph. 


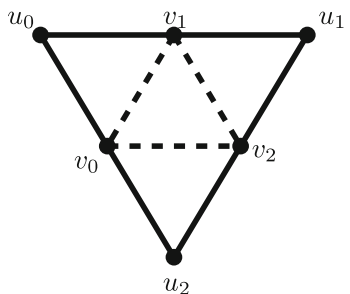

(a)

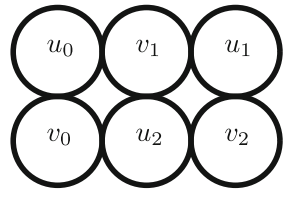

(b)

Fig. 2. (a) The sungraph has no weak unit interval representation, but (b) it has a weak unit disk representation. Near/far edges are indicated with solid/dashed line segments.

Proof. We prove the theorem by induction on the number of edges in a graph. Assume the inductive hypothesis that every 2-reducible graph $G$ with $m$ edges has a weak unit disk representation $I$ with respect to any edge-labeling $\ell$ so that (i) the disks have diameter $t=2$ and (ii) for every edge $(x, y)$ of $G, 1<$ $\|I(x)-I(y)\|<4$. The base case $m=0$ is trivial, so assume that the claim holds for $m>0$ and for any 2-reducible graph $G^{\prime}$ with $m^{\prime}<m$ edges. Now consider an arbitrary 2-reducible graph $G$ with $m$ edges and an arbitrary edge-labeling $\ell$ of $G$.

If $G$ has a vertex of degree 1 , then the desired representation can be constructed by removing the vertex and considering a representation for the resulting graph. Hence, we assume that $G$ has no degree-1 vertices. Then $G$ has a vertex $v$ with exactly two neighbors $u$ and $w$, such that contracting the edge $(u, v)$ results in a 2-reducible graph $G^{\prime}$. We adopt the equivalent convention that, instead of contracting $(u, v)$, we delete $v$ and add the edge $(u, w)$ if it is not already present. Clearly in $G^{\prime}$ the number of edges $m^{\prime}<m$. Thus by the inductive hypothesis, $G^{\prime}$ has a weak unit disk representation $I^{\prime}$ with respect to the edge-labeling $\ell$ restricted to the edges of $G^{\prime}$ (if edge $(u, w)$ does not belong to $G$, give it an arbitrary label). Furthermore, $1<\left\|I^{\prime}(u)-I^{\prime}(w)\right\|<4$. Without loss of generality, assume that $I^{\prime}(u)=(0,0)=p$ (say) and $I^{\prime}(w)=(d, 0)=q$ (say), where $d>0$. Then $1<d<4$. We construct a representation $I$ of $G$ by setting $I(x)=I^{\prime}(x)$ for every vertex $x \neq v$. To compute the value of $I(v)$, consider the following four cases, based on the values of $\ell(u, v)$ and $\ell(w, v)$.

Case 1: $\ell(u, v)=N, \ell(w, v)=N$. If $\ell(u, w)=N$, i.e., the disks for $u$ and $w$ intersect each other, then set $I(v)$ to be the apex of an equilateral triangle with $p q$ as a side. The disk for $v$ then intersect both the disks for $u$ and $w$. Otherwise, if $\ell(u, w)=F$, set $I(v)=(0, d / 2)$. Then $\|I(u)-I(v)\|=\|I(w)-I(v)\|=d / 2$ and since $d<4, d / 2<2$. However since $\ell(u, w)=F$, we have $d>2$; hence $d / 2>1$.

Case 2: $\ell(u, v)=N, \ell(w, v)=F$. Set $I(v)$ to be $(0,-t)$, where $1<t<2$.

Case 3: $\ell(u, v)=F, \ell(w, v)=N$. Set $I(v)$ to be $(0, t)$, where $d+1<t<d+2$. 
Case 4: $\ell(u, v)=F, \ell(w, v)=F$. Set $I(v)$ to be the apex of an isosceles triangle with height $h$ and with $p q$ as the base, where $2<h<3$. Then $\|I(u)-I(v)\|=$ $\|I(w)-I(v)\|=d^{\prime}=\sqrt{h^{2}+(d / 2)^{2}}$. Thus $d^{\prime}>h>2$, so that the disks for $u$ and $w$ do not intersect with the disk for $v$. Furthermore $d<4$ and $h<3$ imply $d^{\prime}<4$.

Series-parallel graphs are defined as the graphs that do not have $K_{4}$ as a minor [8]. Hence by definition, these graphs are closed under edge contraction. It is also well-known that a series-parallel graph is subgraph of a 2-tree, which is 2-reducible, and that every outerplanar graph is a subgraph of a series parallel graph. Thus, by Theorem 1, we have the following corollary.

Corollary 1. Every outerplanar and series-parallel graph is a total weak unit disk graph.

\section{Weak Unit Interval Graph Representations}

In this section we study weak unit interval representability. Given a graph $G=(V, E)$, an edge-labeling $\ell: E \rightarrow\{N, F\}$, and integers $r>0, t \geq 0, G$ is said to be $(r, t)$-threshold-colorable with respect to $\ell$ if there exists a coloring $c: V \rightarrow\{1, \ldots, r\}$ such that for each edge $(u, v) \in E,|c(u)-c(v)| \leq t$ if and only if $\ell(u, v)=N$. The coloring $c$ is known as a threshold-coloring [1]. It is easy to see that threshold-coloring is a special case of weak unit disk representation when restricted to unit interval representation. As defined, threshold coloring requires integer coordinates for the vertices. The next lemma shows that this requirement does not significantly affect the correspondence between the two problems.

Lemma 2. A graph $G$ has a weak unit interval representation for an edgelabeling $\ell$ if and only if $G$ is $(r, t)$-threshold-colorable with respect to $\ell$ for integers $r>0, t \geq 0$.

Proof. We first show that a threshold-coloring $c$ with respect to an edge-labeling $\ell$ yields a weak interval representation for $\ell$. Indeed if $c$ is an $(r, t)$-thresholdcoloring of $G$ with respect to $\ell$, then for each vertex $u$ of $G$, define an interval $I(u)$, which is centered at the point $c(u)$ and has length $t$. Then for any pair of vertices $u, v$ of $G$, the intervals $I(u)$ and $I(v)$ intersect if and only if $|c(u)-c(v)| \leq t$. Since $c$ is a threshold coloring, for any edge $(u, v),|c(u)-c(v)| \leq t$ if and only if $\ell(u, v)=N$. Thus the set of intervals defines a weak interval representation of $G$ for $\ell$.

For the other direction, let $I$ be a weak unit interval representation of $G$ with respect to $\ell$. We can then find a threshold coloring of $G$ from $I$ as follows. Let $c(u)$ be the center of the interval $I(u)$ for each vertex $u$ and let $t$ be the length of the intervals in $I$. Then by definition, for each edge $(u, v)$ of $G,|c(u)-c(v)| \leq t$ if and only if $\ell(u, v)=N$. However, the centers $c(u)$ and the length $t$ are not necessarily integers. We now modify the representation so that the centers $c(u)$ 
and the length $t$ are all integers, while the weak interval representation property is maintained. First increase the length of each interval by some $\epsilon>0$ so that no two intervals intersect each other only at their endpoints. Choose $\epsilon$ so that the intervals have rational lengths. Next perturb the center of each interval by some $\epsilon^{\prime}<\epsilon / 2$ so that each interval is centered at a rational point. Note that for any two intervals $I(u), I(v)$, we have that $I(u)$ and $I(v)$ intersect each other after these modification if and only if they intersected each other before the modification. Finally scale the representation so that the center of each interval is an integer, and the length of the intervals is also an integer. Then the centers of the intervals in the modified representation give a threshold-coloring (although $r$ and $t$ may be large).

Since deciding threshold-colorability is NP-complete [1], so is the recognition problem for weak unit interval graphs.

Lemma 3. It is NP-complete to decide if a graph with an edge-labeling admits a weak unit interval representation

Next, we study weak unit interval representation for some graph classes. We first present a method for representing graphs, which admit a decomposition into a forest and a 2 -independent set. By $G[U]$ we mean the subgraph of $G$ induced by the vertex set $U \subseteq V$. Recall that a subset $\mathcal{I}$ of vertices in a graph $G$ is called independent if $G[\mathcal{I}]$ has no edges. Similarly, $\mathcal{I}$ is called 2-independent if the shortest path in $G$ between any two vertices of $\mathcal{I}$ has length greater than 2. Such decompositions have been applied to other graph coloring problems $[2,3,24]$.

Lemma 4. Suppose $G=(\mathcal{I} \cup \mathcal{F}, E)$ is a graph such that $\mathcal{I}$ is 2-independent, $G[\mathcal{F}]$ is a forest, and $\mathcal{I} \cap \mathcal{F}=\emptyset$. Then $G$ is a total weak unit interval graph.

Proof. We assume that all intervals in the proof are centered at integer coordinates and have length $t=1$. Suppose $\ell: E \rightarrow\{N, F\}$ is an arbitrary edgelabeling. For each $v \in \mathcal{I}$, set $I(v)=0$. Each vertex in $G[\mathcal{F}]$ is assigned a point from $\{-2,-1,1,2\}$ as follows. Choose a component $T$ of $G[\mathcal{F}]$, and select a root vertex $w$ of $T$. If $w$ is far from a neighbor in $\mathcal{I}$, set $I(w)=2$; otherwise, $I(w)=1$. Now perform breadth first search on $T$, assigning an interval for each vertex as it is traversed. When we reach a vertex $u \neq w$, it has one neighbor $x$ in $T$ which has been processed, and at most one neighbor $v \in \mathcal{I}$. If $v$ exists, we choose the interval $I(u)=1$ if $\ell(u, v)=N$, and $I(u)=2$ otherwise. Then, if the label of edge $(u, x)$ is not satisfied by $\|I(u)-I(x)\|$, we multiply $I(u)$ by -1 . If $v$ does not exist, choose $I(u)=1$ or -1 to satisfy the edge $(u, x)$. By repeating the procedure on each component of $G[\mathcal{F}]$, we construct a representation of $G$.

Recall that the maximum average degree of a graph $G$ is the maximum of the average degree of each of its subgraphs $H=\left(V_{H}, E_{H}\right)$, and it is given by $\operatorname{mad}(G)=\max \left(2\left|E_{H}\right| /\left|V_{H}\right|\right)$, where the maximum is taken over all subgraphs of $G$. It is known that every planar graph $G$ of maximum average degree $\operatorname{mad}(G)$ strictly less than $\frac{26}{11}$ can be decomposed into a 2 -independent set and a forest [7]. Hence, 


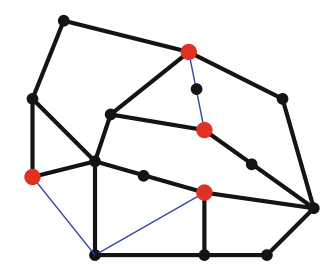

Fig. 3. Decomposition of a graph into a nearly 2-independent set (red vertices) and a forest (black vertices and edges). Thin blue are $\mathcal{I}$-edges. (Color figure online)

Theorem 2. Every planar graph $G$ with $\operatorname{mad}(G)<\frac{26}{11}$ is a total weak unit interval graph.

We also note that a planar graph with girth $g$ satisfies $\operatorname{mad}(G)<\frac{2 g}{g-2}[4]$. Therefore, a planar graph with girth at least 13 always has a weak unit interval representation.

Next we present a generalization of Lemma 4, suitable for graphs which have an independent set that is in some sense nearly 2-independent. The strategy is to delete certain edges so the independent set becomes 2-independent, obtain a unit interval representation using Lemma 4, and then modify it so that it is a representation of the original graph. Formally, let $\mathcal{I}$ be an independent set in a graph $G$. Suppose that for every vertex $v \in \mathcal{I}$, there is at most one vertex $u \in \mathcal{I}$ such that the distance between $v$ and $u$ in $G$ is 2 . Also suppose that there is only one path with two edges connecting $v$ to $u$. Then we call $\mathcal{I}$ nearly 2 -independent. The pair $\{u, v\}$ is called an $\mathcal{I}$-pair, and the edges of the path $(u, x, v)$ connecting $u$ and $v$ are called $\mathcal{I}$-edges, which are associated with the $\mathcal{I}$-pair $\{u, v\}$; see Fig. 3 .

Lemma 5. Let $G=(\mathcal{I} \cup \mathcal{F}, E)$ be a graph, where $\mathcal{I}$ is a nearly 2-independent set, $G[\mathcal{F}]$ is a forest and $\mathcal{I} \cap \mathcal{F}=\emptyset$. Then $G$ has a total weak unit interval graph.

Proof. Assume that all intervals in the proof are centered at integer coordinates and have size $t=3$. Suppose that $\ell: E \rightarrow\{N, F\}$ is an arbitrary edge-labeling of $G$. Let $E^{\prime} \subseteq E$ be a set such that for each $\mathcal{I}$-pair $\{u, v\}$, exactly one of the $\mathcal{I}$-edges associated with $\{u, v\}$ belongs to $E^{\prime}$. Let $G^{\prime}=\left(V, E-E^{\prime}\right)$. Then clearly $\mathcal{I}$ is 2 -independent in $G^{\prime}$ and $G^{\prime}[\mathcal{F}]$ is a forest; by Lemma 4 , there exists a weak unit interval representation $I^{\prime}$ of $G^{\prime}$ for $\ell$.

We now modify $I^{\prime}$ to construct a weak unit interval representation $I$ of $G$ with respect to $\ell$. First, for each vertex $v \in V$, set $I(v)=0$ if $I^{\prime}(v)=0$, $I(v)=2$ if $I^{\prime}(v)=1$, and $I(v)=5$ if $I^{\prime}(v)=2$ (if $I^{\prime}(v)$ is negative, do the same but set $I(v)$ negative). It is clear that $I$ is a weak unit interval representation of $G^{\prime}$. Now, let $(x, y) \in E^{\prime}$. One of these vertices, say $x$, is in $\mathcal{I}$ so $I(x)=0$, and $I(y) \in\{-5,-2,2,5\}$. Without loss of generality assume that $I(y)>0$; the case where $I(y)<0$ is symmetric. Now it is possible that $\ell(x, y)=N$ but $\|I(x)-I(y)\|>3$ or that $\ell(x, y)=F$ but $\|I(x)-I(y)\| \leq 3$. In the first case, we must have $I(y)=5$. We modify $I$ so that $I(x)=1$ and $I(y)=4$. Note that $y$ is still near to vertices with intervals centered at 2 or 5 , and far from vertices 
with intervals centered at less than 1 . Similarly, $x$ is still close to the intervals at $-2,0$, or 2 , but far from -5 and 5 . Thus all the edges of $E-E^{\prime}$ are satisfied by the modification of $I$, and additionally the edge $(x, y)$ is satisfied. In the second case, we have $I(y)=2$. We modify $I$ so that $I(x)=-1$ and $I(y)=3$. As before, no edges which disagreed with the edge-labeling still disagree with the edge-labeling.

Since $\mathcal{I}$ is nearly 2 -independent, our modifications to the representation $I$ will not affect non-local vertices, as every vertex in $\mathcal{I}$ is adjacent to at most one edge of $E^{\prime}$.

\section{Weak Unit Interval Representation of Outerplanar Graphs}

It is known [1] that some outerplanar graphs containing triangles are not total weak unit interval graphs, e.g., the sungraph in Fig. 2. Hence, we study weak unit interval representability of triangle-free outerplanar graphs. We start with a claim for girth 5 .

Lemma 6. An outerplanar graph with girth 5 is a total weak unit interval graph.

Proof. We prove that girth-5 outerplanar graphs may be decomposed into a forest and a 2-independent set using induction on the number of internal faces. The result will follow from Lemma 4. The claim is trivial for a single internal face, so assume that it is true for all girth-5 outerplanar graphs with $k \geq 1$ internal faces. Let $G$ be a girth-5 outerplanar graph with $k+1$ internal faces. Since $G$ is outerplanar, it must have at least one face $f=\left(v_{1}, \ldots, v_{l}\right), l \geq 5$, such that every vertex of $f$ except $v_{1}, v_{l}$ is of degree 2. Consider the graph $G^{\prime}$ obtained by deleting $v_{2}, \ldots, v_{l-1}$. The vertices of $G^{\prime}$ have a decomposition into a 2 -independent set $\mathcal{I}$ and a set $\mathcal{F}$ such that $G^{\prime}[\mathcal{F}]$ is a forest. Now we will add the vertices $v_{2}, \ldots, v_{l-1}$ to either $\mathcal{I}$ or $\mathcal{F}$ so that $\mathcal{I}$ is a 2 -independent set in $G$, and $G[\mathcal{F}]$ is a forest. If either of $v_{1}, v_{l}$ belongs to $\mathcal{I}$, then add all the remaining vertices to $\mathcal{F}$. Otherwise, add $v_{3}$ to $\mathcal{I}$ and the rest to $\mathcal{F}$. Since $v_{1}, v_{l}$ are not in $\mathcal{I}, v_{3}$ has distance at least 3 from any other element of $\mathcal{I}$.

Next our goal is to show that a triangle-free outerplanar graph $G$ always has a weak unit interval representation for any edge-labeling. We assume that all intervals are centered at integer coordinates and we use intervals of size $t=2$. Our strategy is to find a representation of $G$ by a traversal in a depth-first search manner of its weak dual graph $G^{*}$ (the planar dual minus the outerface). We find intervals for all the vertices in each interior face of $G$ as it is traversed in $G^{*}$. Since we are considering triangle-free graphs, this implies that we take an induced path $P_{n}=\left(u_{1}, u_{2}, \ldots, u_{n}\right)$ of $G, n \geq 4$, where the two end vertices $u_{1}$ and $u_{n}$ are already processed and we need to assign unit intervals to the internal vertices $u_{2}, \ldots, u_{n-1}$ of $P_{n}$. Note that this path $P_{n}$ along with the edge $(u, v)$ forms an internal face of $G$. We additionally maintain the invariant in our representation that for each edge $(u, v)$ of $G,\|I(u)-I(v)\| \leq 6$. For a particular edge-labeling $\ell$ of $P_{n}=\left(u_{1}, \ldots, u_{n}\right)$, call a pair of coordinates $x, y$ feasible if there is a weak unit interval representation $I$ of $P_{n}$ for $\ell$ with $t=2$, where 
$I\left(u_{1}\right)=x, I\left(u_{n}\right)=y$, and for any $i \in\{1, \ldots, n-1\},\left\|I\left(u_{i}\right)-I\left(u_{i+1}\right)\right\| \leq 6$. We first need the following three claims.

Claim 1. For any value of $x \in\{2,3,-2,-3\}$, the pair $0, x$ is feasible for any edge-labeling $\ell$ of $P_{3}=\left(u_{1}, u_{2}, u_{3}\right)$.

Proof. Without loss of generality, we may assume that $x>0$. We compute a desired weak unit interval representation $I$ with $t=2$ for $P_{3}$ with respect to $\ell$ as follows. Assign $I\left(u_{1}\right)=0$ and $I\left(u_{3}\right)=x$. Assign $I\left(u_{2}\right)$ in such a way that $\left|I\left(u_{2}\right)\right|=2$ if $\ell\left(u_{1}, u_{2}\right)=N$, and $\left|I\left(u_{2}\right)\right|=3$ if $\ell\left(u_{1}, u_{2}\right)=F$. Then choose the sign of $I\left(u_{2}\right)$ to be the same as $I\left(u_{3}\right)$ if $\ell\left(u_{2}, u_{3}\right)=N$, and the opposite of $I\left(u_{3}\right)$ if $\ell\left(u_{2}, u_{3}\right)=F$.

Claim 2. For any edge-labeling of $P_{3}=\left(u_{1}, u_{2}, u_{3}\right)$, either 0,4 or 0,6 are feasible.

Proof. We compute a desired weak unit interval representation $I$ with $t=2$ for $\ell$ as follows. If $\ell\left(u_{1}, u_{2}\right)=l\left(u_{2}, u_{3}\right)=N$, then $I\left(u_{1}\right)=0, I\left(u_{2}\right)=2$, and $I\left(u_{3}\right)=4$. Otherwise, assign $I\left(u_{1}\right)=0, I\left(u_{3}\right)=6$, and $I\left(u_{2}\right)=2,3$ or 4 when $\left(l\left(u_{1}, u_{2}\right), l\left(u_{2}, u_{3}\right)\right)$ have values $(N, F),(F, F)$, and $(F, N)$, respectively.

Claim 3. For any integer value of $x \in[-6,6]$, the pair $0, x$ is feasible for any edge-labeling of $P_{n}=\left(u_{1}, u_{2}, \ldots, u_{n}\right), n \geq 4$.

Proof. Without loss of generality, let $x \geq 0$. Consider first the case for $n=4$. Take a particular edge-labeling $\ell$ of $P_{4}$. For any integer value of $0 \leq x \leq 5$, there is at least one number $y \in\{2,3,-2,-3\}$ and at least one number $z \in$ $\{2,3,-2,-3\}$ such that $|x-y| \leq 2$ and $2<|x-z| \leq 6$. In particular, it suffices to choose for $x=0, y=2, z=3$; for $x=1,2,3,4, y=2, z=-2$ and for $x=5, y=3, z=2$. Thus if $0 \leq I\left(u_{4}\right) \leq 5$, and regardless of whether $\ell\left(u_{3}, u_{4}\right)$ is $N$ or $F$, one can choose a value for $I\left(u_{3}\right)$ from $\{2,3,-2,-3\}$ respecting both the edge-labeling of $\left(u_{3}, u_{4}\right)$ and the property that $\left\|I\left(u_{3}\right)-I\left(u_{4}\right)\right\| \leq 6$. Then by Claim 1, 0 and $x$ is feasible for the edge-labeling $\ell$ of $P_{4}$. A similar argument shows that if $\ell\left(u_{3}, u_{4}\right)=F$, then 0 and $x=6$ is feasible. On the other hand, if $x=6$ and $\ell\left(u_{3}, u_{4}\right)=N$, then both 4 and 6 are valid choices for $I\left(u_{3}\right)$. By Claim 2, 0 and 6 is feasible for any edge-labeling $\ell$ of $P_{4}$.

Consider now the case with $n>4$. Then assign coordinates $I\left(u_{1}\right)=0$, $I\left(u_{n}\right)=x$ and for $i \in\{n-1, \ldots, 4\}$, assign $I\left(u_{i}\right) \in[-6,6]$ such that it respects both $\ell\left(u_{i}, u_{i+1}\right)$ and the property that $\left\|I\left(u_{i}\right)-I\left(u_{i+1}\right)\right\| \leq 6$. Then a similar argument as that for $n=4$ can be used to extend this representation to $u_{2}$ and $u_{3}$.

The next corollary immediately follows from Claim 3.

Corollary 2. Any pair $x, y$ with $|x-y| \leq 6$, is feasible for any edge-labeling of $P_{n}=\left(u_{1}, u_{2}, \ldots, u_{n}\right), n \geq 4$.

Theorem 3. Every triangle-free outerplanar graph is a total weak unit interval graph. 
Proof. If $G$ is not 2-connected, we augment it in the following way. Let $v$ be a cut vertex of $G$ and let $H_{1}, \ldots, H_{k}$ be the 2-connected components of $G$ containing $v$. For $i \in\{1, \ldots, k-1\}$, let $u$ be a neighbor of $v$ in $H_{i}$, and $w$ be a neighbor of $v$ in $H_{i+1}$. Add the path $(u, x, w)$, where $x$ is a new vertex. Clearly, any weak unit interval representation of the new 2-connected graph is also a weak unit interval representation of $G$, and the new graph is outerplanar with girth 4 .

Now let $G$ be a 2-connected triangle-free outerplanar graph with $n>4$ vertices embedded in the plane with every vertex on the outerface, and let $\ell$ be an arbitrary edge-labeling of $G$. We next compute a weak unit interval representation of $G$ for $\ell$. The proof is by induction on the number of vertices in $G$, with the $n$-vertex cycle as a base case. Assume the inductive hypothesis that every triangle-free outerplanar graph with fewer than $n$ vertices is a total weak unit interval graph. Further, assume that for such a graph $G^{\prime}$ with any edgelabeling $\ell^{\prime}$, there is a weak unit interval representation of $G^{\prime}$ for $\ell^{\prime}$ where any two neighbor vertices $u$ and $v$ satisfy $\|I(u)-I(v)\| \leq 6$. Clearly if $G$ has at least two cycles, then $G$ has a path $P_{k}=\left(u_{1}, \ldots, u_{k}\right), k \geq 4$ with $\operatorname{deg}\left(u_{i}\right)=2$ for some $1<i<k$. The theorem follows from the inductive hypothesis and Corollary 2 .

\section{Planar Graphs without Weak Unit Interval Representations}

Planar graphs with high edge density may not have weak unit interval representations. First we prove the result for a wheel graph, defined as $W_{n}, n \geq 4$, formed by adding an edge from a vertex $v_{1}$ to every vertex of an $(n-1)$-cycle $\left(v_{2}, \ldots, v_{n}, v_{2}\right)$.

Lemma 7. A wheel graph is not a total weak unit interval graph.

Proof. Define an edge-labeling $\ell$ of $W_{n}$ by $\ell\left(v_{2}, v_{n}\right)=F, \ell\left(v_{1}, v_{i}\right)=F$ for $3 \leq i \leq n-1$, and every other edge labeled $N$; see Fig.4(a). Suppose $I$ is a weak unit interval representation of $W_{n}$ with respect to $\ell$. Since only one edge of the triangle $\left(v_{1}, v_{2}, v_{n}\right)$ is far, $I\left(v_{1}\right) \neq I\left(v_{2}\right)$, hence assume that $I\left(v_{1}\right)<I\left(v_{2}\right)$. For $3 \leq i \leq n$, if $I\left(v_{i-1}\right)>I\left(v_{1}\right)$, we have $I\left(v_{i}\right)>I\left(v_{1}\right)$, since $\ell\left(v_{i-1}, v_{i}\right)=N$ and either $\ell\left(v_{1}, v_{i-1}\right)$ or $\ell\left(v_{1}, v_{i}\right)$ is $F$. Then $I\left(v_{1}\right)<I\left(v_{2}\right) \leq I\left(v_{1}\right)+1$, and $I\left(v_{1}\right)<I\left(v_{n}\right) \leq I\left(v_{1}\right)+1$, contradicting that $\ell\left(v_{2}, v_{n}\right)=F$ and $I$ is weak interval representation.

Using Lemma 7, it is easy to see that any maximal planar graph with $|V| \geq 4$ is not a weak unit interval graph. Indeed, consider such a graph $G=(V, E)$ and a vertex $v \in V$; the neighborhood $N(v)=\{u \mid(v, u) \in E\}$ together with $v$ induces a wheel subgraph. The observation leads to the following theorem.

Theorem 4. Any planar graph $G$ with $\operatorname{mad}(G) \geq \frac{11}{2}$ is not a total weak unit interval graph.

Proof. To prove the claim, we show that a total weak unit interval planar graph has at most $\lfloor 11|V| / 4\rfloor-6$ edges.

Consider a vertex $v$ of a weak unit interval planar graph $G=(V, E)$ and assume it is embedded in the plane. The neighborhood of $v$ is acyclic; otherwise 


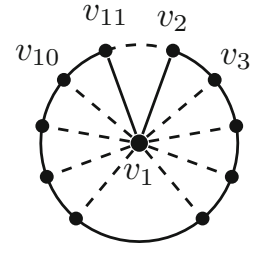

(a)

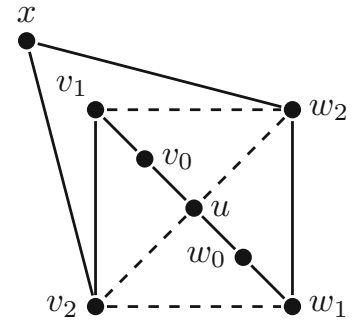

(b)

Fig. 4. (a) A wheel graph $W_{11}$ with an edge-labeling, that has no weak unit interval representation. (b) A girth-4 graph with an edge-labeling, that has no weak unit interval representation.

$v$ and its neighborhood induce a wheel, which by Lemma 7 is not a weak unit interval graph. Thus the number of edges between any two neighbors of $v$ is at $\operatorname{most} \operatorname{deg}(v)-1$, where $\operatorname{deg}(v)$ is the degree of $v$. Denote the number for a vertex $v$ by $s(v)$. Consider the sum, $S=\sum_{v} s(v)$, taken over all vertices of $G$. It is easy to see that $S \leq 2|E|-|V|$.

Let $T$ and $\bar{T}$ be the sets of triangular and non-triangular faces in an embedding of $G$. For each triangle $x \in T$ each of the edges in $x$ is counted once in $S$. Thus, $2|E|-|V| \geq 3|T| \Rightarrow|T| \leq(2|E|-|V|) / 3$. Counting both sides of the edges we get $2|E| \geq 3|T|+4|\bar{T}| \Rightarrow|T|+|\bar{T}| \leq(2|E|+|T|) / 4 \leq(8|E|-|V|) / 12$, since $|T| \leq(2|E|-|V|) / 3$. Thus, from Euler's formula $|V|-|E|+|T|+|\bar{T}|=2$, we have $|V|-|E|+(8|E|-|V|) / 12 \geq 2 \Rightarrow|E| \leq 11|V| / 4-6$.

In [1] all examples of graphs without threshold-coloring (and thus not total weak unit interval graphs) have girth 3 . We strengthen the bound by proving the following.

Lemma 8. There exist planar girth-4 graphs that are not total weak unit interval graphs.

Proof. Consider the graph in Fig.4(b) with the given edge-labeling. Suppose there exists a weak unit interval representation $I$. Without loss of generality suppose that $I\left(w_{2}\right)>I(u)$. Let us consider two cases. First, suppose $I\left(v_{2}\right)<$ $I(u)$. Since the edges $\left(u, v_{2}\right)$ and $\left(u, w_{2}\right)$ are labeled $F$, it must be that $I\left(v_{2}\right)<$ $I(u)-1$ and $I(u)+1<I\left(w_{2}\right)$. Then vertex $x$ must be represented by an interval near to both of these, which is impossible since $\left\|I\left(v_{2}\right)-I\left(w_{2}\right)\right\|>2$.

Otherwise $I\left(v_{2}\right)>I(u)$. Then $I\left(v_{1}\right) \geq I\left(v_{2}\right)-1>I(u)$, and $I(u)<I\left(w_{2}\right)$ implies that $I\left(v_{1}\right)<I\left(w_{2}\right)$. Similarly, $I\left(w_{1}\right)<I\left(v_{2}\right)$. Now, either $I\left(w_{2}\right) \leq I\left(v_{2}\right)$, or $I\left(v_{2}\right)<I\left(w_{2}\right)$. In the first case, $w_{2}$ is near to $v_{1}$ since $I\left(v_{1}\right)<I\left(w_{2}\right) \leq I\left(v_{2}\right)$ and $\left\|I\left(v_{1}\right)-I\left(v_{2}\right)\right\| \leq 1$. The second case leads to a similar contradiction. 


\section{Conclusion and Open Problems}

In this paper we introduced the concept of weak intersection representation of graphs and studied representations of planar graphs with unit disks and unit intervals. A natural future direction is to consider weak intersection representations for other graph classes and/or with different geometric objects. Next we list several interesting open problems.

1. Deciding whether a graph has a weak unit disk (interval) representation for a given edge-labeling is NP-hard. However, the problem of deciding whether a graph is a total weak unit disk (interval) graph is open, and it remains open when restricted to planar graphs. Note that the class of total weak unit disk (interval) planar graphs is not closed under taking minors, as subdividing each edge of a planar graph three times results in a planar graph with girth at least 10 , which is a total weak unit interval graph.

2. Tightening the lower and upper bounds for maximum average degree of total weak unit interval planar graphs, given in Theorems 2 and 4, is a challenging open problem. Based on extensive computer experiments, we conjecture that there are no total weak unit interval graphs with more than $2|V|-3$ edges.

3. We considered planar graphs, but little is known for general graphs. In particular, it would be interesting to find out whether the edge density of total weak unit disk (interval) graphs is always bounded by a constant.

4. We proved that a graph has a weak unit interval representation for an edgelabeling $\ell$ if and only if it is $(r, t)$-threshold-colorable with respect to $\ell$ for integers $r>0, t \geq 0$, but in the proof, the values of $r$ and $t$ can be arbitrarily large. It would be interesting to bound the values of $r$ and $t$ for any $n$-vertex graph.

Acknowledgments. We thank Michalis Bekos, Gasper Fijavz, and Michael Kaufmann for productive discussions about several variants of these problems.

\section{References}

1. Alam, M.J., Chaplick, S., Fijavž, G., Kaufmann, M., Kobourov, S.G., Pupyrev, S.: Threshold-coloring and unit-cube contact representation of graphs. In: Brandstädt, A., Jansen, K., Reischuk, R. (eds.) WG 2013. LNCS, vol. 8165, pp. 26-37. Springer, Heidelberg (2013)

2. Alam, M.J., Kobourov, S.G., Pupyrev, S., Toeniskoetter, J.: Happy edges: threshold-coloring of regular lattices. In: Ferro, A., Luccio, F., Widmayer, P. (eds.) FUN 2014. LNCS, vol. 8496, pp. 28-39. Springer, Heidelberg (2014)

3. Albertson, M.O., Chappell, G.G., Kierstead, H.A., Kündgen, A., Ramamurthi, R.: Coloring with no 2-colored P4. Electron. J. Combin. 11(1), R26 (2004)

4. Borodin, O., Kostochka, A., Nešetřil, J., Raspaud, A., Sopena, E.: On the maximum average degree and the oriented chromatic number of a graph. Dis. Math. 206(1), 77-89 (1999) 
5. Bremner, D., Evans, W., Frati, F., Heyer, L., Kobourov, S.G., Lenhart, W.J., Liotta, G., Rappaport, D., Whitesides, S.H.: On representing graphs by touching cuboids. In: Didimo, W., Patrignani, M. (eds.) GD 2012. LNCS, vol. 7704, pp. 187-198. Springer, Heidelberg (2013)

6. Breu, H., Kirkpatrick, D.G.: Unit disk graph recognition is NP-hard. Comput. Geom. 9(1), 3-24 (1998)

7. Bu, Y., Cranston, D.W., Montassier, M., Raspaud, A., Wang, W.: Star coloring of sparse graphs. J. Graph. Theory 62(3), 201-219 (2009)

8. Duffin, R.: Topology of series-parallel networks. J. Math. Anal. Appl. 10, 303-318 (1965)

9. Eggleton, R., Erdös, P., Skilton, D.: Colouring the real line. J. Comb. Theory, Ser. B 39(1), 86-100 (1985)

10. Evans, W., Gansner, E.R., Kaufmann, M., Liotta, G., Meijer, H., Spillner, A.: Approximate proximity drawings. In: Speckmann, B. (ed.) GD 2011. LNCS, vol. 7034, pp. 166-178. Springer, Heidelberg (2011)

11. Herrera de Fegueiredo, C.M., Meidanis, J., Picinin de Mello, C.: A linear-time algorithm for proper interval graph recognition. Inf. Process. Lett. 56(3), 179-184 (1995)

12. Ferrara, M., Kohayakawa, Y., Rödl, V.: Distance graphs on the integers. Comb. Probab. Comput. 14(1), 107-131 (2005)

13. Gabriel, K.R., Sokal, R.R.: A new statistical approach to geographic variation analysis. Syst. Biol. 18(3), 259-278 (1969)

14. Golumbic, M.C., Kaplan, H., Shamir, R.: Graph sandwich problems. J. Algorithms 19(3), 449-473 (1995)

15. Hale, W.K.: Frequency assignment: theory and applications. Proc. IEEE 68(12), 1497-1514 (1980)

16. Hammer, P.L., Peled, U.N., Sun, X.: Difference graphs. Dis. App. Math. 28(1), 35-44 (1990)

17. Hliněnỳ, P., Kratochvíl, J.: Representing graphs by disks and balls (a survey of recognition-complexity results). Discrete Math. 229(1), 101-124 (2001)

18. Kleist, L., Rahman, B.: Unit contact representations of grid subgraphs with regular polytopes in 2D and 3D. In: Duncan, C., Symvonis, A. (eds.) GD 2014. LNCS, vol. 8871, pp. 137-148. Springer, Heidelberg (2014)

19. Kuhn, F., Wattenhofer, R., Zollinger, A.: Ad hoc networks beyond unit disk graphs. Wireless Netw. 14(5), 715-729 (2008)

20. Liotta, G.: Proximity drawings. In: Tamassia, R. (ed.) Handbook of Graph Drawing and Visualization. Chapman \& Hall/CRC, Boca Raton (2007)

21. Mahadev, N.V., Peled, U.N.: Threshold Graphs and Related Topics. North Holland, Amsterdam (1995)

22. McDiarmid, C., Müller, T.: Integer realizations of disk and segment graphs. J. Comb. Theory, Ser. B 103(1), 114-143 (2013)

23. Thomassen, C.: Decomposing a planar graph into degenerate graphs. J. Comb. Theory, Ser. B 65(2), 305-314 (1995)

24. Timmons, C.: Star coloring high girth planar graphs. Electron. J. Comb. 15(1), R124 (2008)

25. Wiegers, M.: Recognizing outerplanar graphs in linear time. In: Tinhofer, G., Schmidt, G. (eds.) WG 1986. LNCS, vol. 246, pp. 165-176. Springer, Heidelberg (1987) 